\title{
From Information Retrieval to Information Extraction
}

\author{
David Milward and James Thomas \\ SRI International \\ Suite 23 Millers Yard \\ Mill Lane \\ Cambridge \\ CB2 1RQ \\ milward@cam.sri.com, jrt1003@cam.sri.com
}

\begin{abstract}
This paper describes a system which enables users to create on-the-fly queries which involve not just keywords, but also sortal constraints and linguistic constraints. The user can specify how the results should be presented e.g. in terms of links to documents, or as table entries. The aim is to bridge the gap between keyword based Information Retrieval and pattern based Information Extraction.
\end{abstract}

\section{Introduction}

The amount of information available electronically in a free-text format is ever increasing. People require tools that give them the best possible answer to their queries even when a full answer may not be available.

Current web Information Retrieval (IR) engines standardly retrieve URLs to whole documents, and typical user queries are just an unordered set of keywords. This is robust and allows unrestricted queries, but precision can be poor, and the output is not particularly convenient for many queries. For example, if we are interested in e.g. companies associated with Mr. Jones, we are likely to prefer an output in terms of an alphabetically ordered list of companies (with links to the sentences in which they appear) rather than a list of URLs.

Information Extraction (IE) systems provide better presentation of results (e.g. tables, database records etc.), and tend to include more precise searches which depend upon not just finding keywords but finding them in particular positions or in particular grammatical relationships. However, current IE systems typically require queries to be programmed beforehand, so are suitable only for cases where the same query is to be used time and again e.g. in the processing of a newsfeed.
Customisation of an IE system involves two major tasks:

- adapting the information extraction system to a new domain, e.g. adding vocabulary, new grammatical constructions and domain ontology.

- creating new patterns for extraction in the new domain.

Customising current IE systems is thus expensive. A domain expert and a linguist with knowledge of the IE system will generally be required to specify the task, manually mark-up or categorise domain data, perhaps build a domain ontology, write extraction patterns and fine-tune them for the best compromise of precision and recall.

There are thus pressing reasons for automating the customisation task and reducing the involvement of linguists as much as possible. Several methods of automatically inferring patterns and semantic dictionaries from annotated texts have been suggested, e.g. (Riloff, 1993; Riloff, 1996; Sonderland et al., 1995; Yangarber and Grishman, 1997). Pierce and Cardie (Cardie and Pierce, 1998) suggest that the use of annotated texts can be replaced by an interactive approach in which an end-user assists the system in bootstrapping patterns from a set of example results. However even this method is ill suited to one-off queries, where the user is unlikely to start from example results, but needs to be able to interactively home in on a set of answers.

In this paper we describe a system which enables users to build advanced on-the-fly queries. The interface is intended to be intuitive for users already familiar with keyword IR but adds the extra functionality of sortal, linguistic and positional constraints that are more common in IE. Adding extra linguistic constraints may improve precision, and cause a drop in recall, in exactly the same way as adding an extra non-optional keyword.

The approach is less ambitious than providing full Natural Language querying, but allows 
professional users of search technology (e.g. analysts or research scientists) to get most of the advantages of IE without having to program patterns. We assume that retraining of linguistic components (such as the tokeniser and tagger) for a new domain will still be performed by a linguist/programmer, and that end-users will be supplied with libraries of useful sorts such as person names, companies, locations, protein names etc. which are appropriate for their domain.

\section{IR and NLP}

Our key interest in this work was to provide a system which allowed users to get answers: not just documents or sub-documents. We have not addressed the question of whether or not these techniques would also be useful for more traditional IR in the sense of finding the most relevant document for a particular query. There is some potential since there are extra options to refine or expand a query e.g. using sortal constraints such as company and location, and restrictive constraints such as subject_of or same sentence. Since the linguistic constraints are under user control the query is more likely to be accurate than in systems where linguistic constraints are derived from a natural language query (though at the expense of usability).

The system was designed to deal with multiple answer queries such as "which protein interacts with TAF-2?". This differs somewhat from the TREC question answering track ((TREC), 2000), where the emphasis is on questions which have a single answer, and systems attempt to provide the most relevant sub-document. To attempt the TREC task we would need to extend the system with a relevance weighting mechanism, and provide further techniques for query expansion. We would then expect the system to do well, since (Srihari and $\mathrm{Li}, 1999$ ) show that the use of sortal constraints such as company, location and time plus constraints such as same sentence give good results, even with a relatively simple ranking mechanism.

How much extra cost is involved in using linguistic information? There is obviously some initial cost in parsing and analysing the texts, however this can largely be hidden by preprocessing the documents. In addition there is a space cost in having a much larger index: this is necessary since we are keeping more information about the document's structure. Finally, there is the cost of evaluating more complex constraints. The cost of using sortal constraints is negligible: we can index them in exactly the same way as words. However, i1: $\mathrm{P}$

i2: a

i3: b

$i 4: i 1(i 2, i 3)$

Figure 1: Distributed representation of $P(a, b)$

relational constraints such as same sentence do introduce extra processing. The figures we present at the end of this paper show that this first implementation of the system is fast enough to be usable for some real applications (and very fast by Information Extraction standards), but is not yet in the same league as standard IR engines.

\section{Highlight}

Highlight (Thomas et al., 2000) is a generalpurpose IE engine for use in commercial applications. The work described in the current paper extends Highlight by building an interface on top of it and replacing the internal representation of linguistically analysed texts with a representation based on distributed representations c.f. (Milward, 2000). Demos of the Highlight System are accessible from the SRI Cambridge web site at http://www.cam.sri.com

\subsection{Distributed Representation}

The inspiration for the approach taken here was the work in (Milward, 2000) on interpretation of dialogue utterances. This uses distributed semantic representations (based on indexing individual parts of a semantic structure) to encode both full and partial semantic analyses. For example, the logical form $\mathbf{P}(\mathrm{a}, \mathrm{b})$ is represented by the set of constraints given in Figure 1.

This approach aims to combine the advantages of shallow pattern-matching approaches (which are fast and robust) with those of deeper analysis which tends to do better for phenomena which involve scope. For example, consider the utterance "The 2.10 flight to Boston will not stop at New York". A simple pattern matching approach looking for [flight] to [location] via [location] would extract the wrong information since it has no notion of the scope of the negation.

In this work we take the same idea, but apply it to Information Extraction. Sets of indexed constraints (which are themselves partial descriptions of semantic structure) are used directly as search expressions. Since the indexed constraints can encode full semantic structures, the search terms can be more or less specific: we can start with just constraints on lexical items, and then build up to a full semantic structure by adding structural con- 


\begin{tabular}{ll} 
obligatory & \multicolumn{1}{c}{ optional } \\
\hline$I: J(K, L)$, & (1) $K>H, H: M(N) \quad$ (2) $K: M(N)$ \\
$J: a t$, & \\
$M: l e a v e$, & \\
$L: T$, & \\
time (T) &
\end{tabular}

Figure 2: Extracting a departure time

straints.

Search is now a question of expressing the appropriate constraints on the information to be extracted. For example, Figure 2 is a sketch rule to extract departure time from a sentence such as "I leave at $3 \mathrm{pm}$." The rule subsumes a range of rules which vary in the specificity with which they apply. For example, applying only the obligatory conditions will result in the extraction of $3 \mathrm{pm}$ as departure time from sentences such as "I leave Cambridge to arrive at $3 \mathrm{pm}$ " since in the obligatory component there is no restriction on $M$ with respect to $T$, i.e. the leave event and the time. Adding the optional constraints in (1) gives us the restriction that the leaving must be dominated by the preposition at, e.g. "I leave from the station at $3 \mathrm{pm}, "$ and (2) requires immediate dominance: "I leave at $3 \mathrm{pm}$." This ranges from pattern matching to almost full semantics. Any particular application of a rule can be scored according to the number of optional conditions which are fulfilled, thereby using linguistic constraints if they are available.

In practice, the system described here departs from this approach in several respects. Firstly, we were working with the pre-existing Highlight system which uses shallow syntactic processing based on cascaded patterns (similar to (Hobbs et al., 1996)). Deriving semantic relationships from this is not particularly reliable, so it is preferable to use search terms which rely more on positional clues. We therefore used a distributed syntactic representation to provide more reliable syntactic constraints (e.g. head word of, subject of, same sentence) augmented by positional constraints (e.g. precedes, immediately precedes). Secondly, we wanted an intuitive user interface so constraints such as 'dominates' were not appropriate.

\subsection{User Interface}

Given the underlying representation described above, the user's task in building a query is to propose a set of constraints which can be matched against the representation of a text or set of texts. The interface attempts to preserve the convenience of keyword based IR but also enable more refined searches, and control over the presentation of results. Keyword based search is a special case where the user specifies one or more keywords which they want to find in a document. However, users can also specify that they want to find a class of item (e.g. companies) and refine the search for items within the same sentence, not just the same document.

For example, to find 'Esso' and a location in the same sentence we need the set of constraints given below:

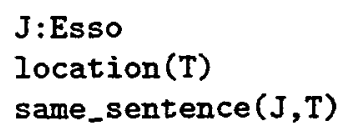

The interface emphasises the items the user wants to search for. Consider Figure 3. The user is looking for two items, the first including the word Esso (this could be e.g. "Esso Corp.", "Esso Holdings" etc.), and the second item of sort 'location'. The effect of pressing 'Add' is to include the positional constraint that the two items must appear in the same sentence. In later parts of this paper we provide examples of more sophisticated queries which imitate what is currently achieved by pattern matching in more standard IE systems (e.g. Fastus (Hobbs et al., 1996)).

In our approach IE is a seamless extension of IR. This can be contrasted with some more typical ways of tying together IE and IR by performing an IR search followed by IE. In that approach, IR is used first to select a limited set of documents, then IE is applied. This is fine if the queries and the keywords are fixed (and appropriate for each other). It is not ideal otherwise. For example, suppose you are interested in Esso's profits. To achieve your query you might use IR to find documents containing Esso, then use an IE system which has been customised to look for company profits. However, some of the results are likely to be unexpected, for example, you would obtain Shell's profits if there were a document describing Shell's profits which just happens to mention Esso in passing.

Items can be constrained to have a particular head word, to include a particular word or to be of 


\section{Intelligent Search and Extraction of Information}

Expert Mode
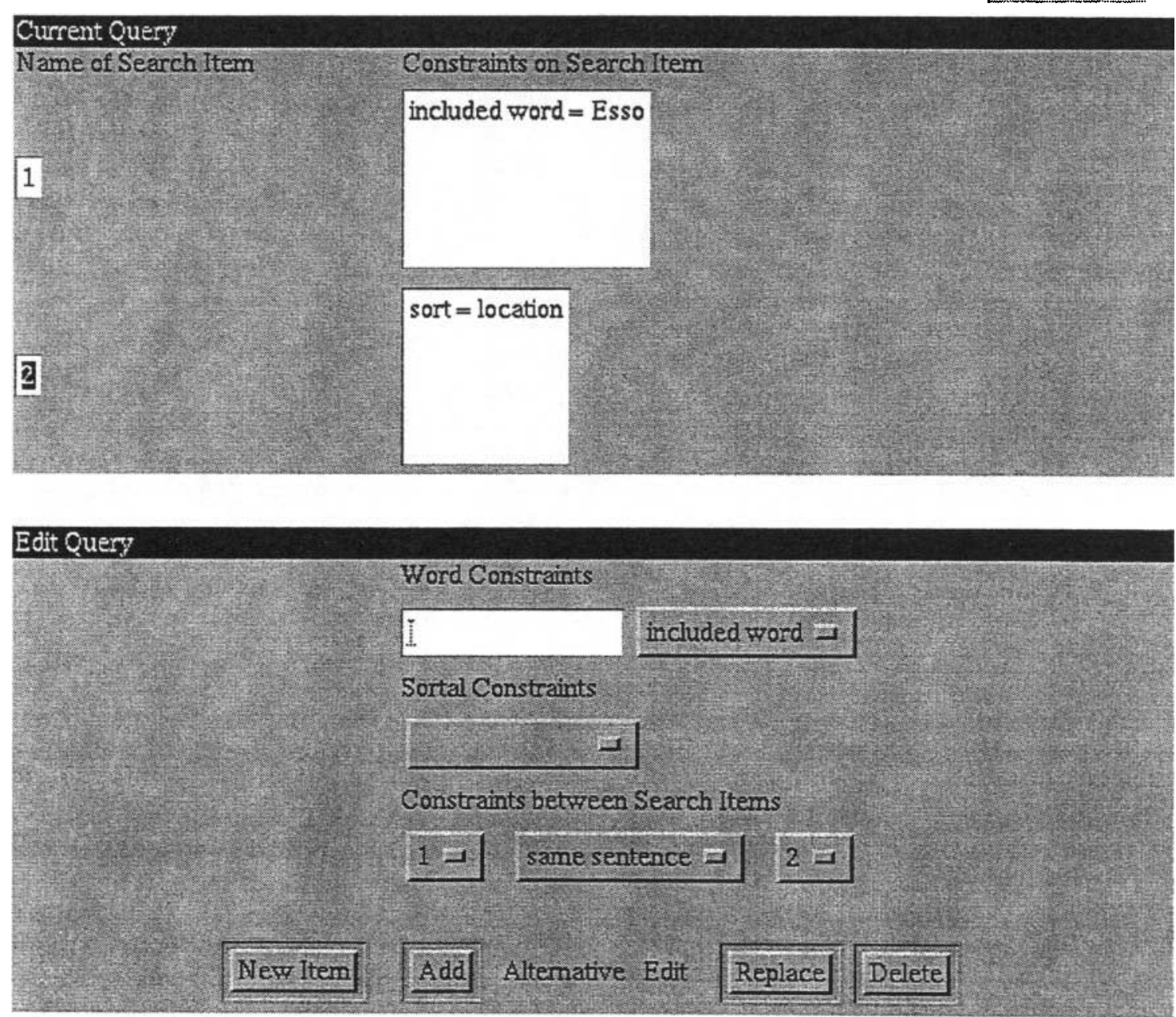

Figure 3: User interface 
a particular sort. Multiple constraints on a single item are possible, as shown in Figure 5. Positional constraints can include any kind of inter-item constraints e.g. precedes, same sentence. There are further option buttons which concern the files to be queried and the layout of the output. The default is to provide a table which includes each item found plus the sentence in which it appears in the document.

Two levels of user expertise (novice and expert) allow more or less control over the technical details of the query. Expert mode (accessed by clicking the button at the top right) looks much the same but has facilities such as altering the template output, naming the items and more options on the pull-down menus. For instance, in expert mode the user may specify which items are optional in the query, and what their syntactic class might be. There are also additional extra parameters for the expert user for the output templates.

A typical user query is given in Figure 4. Here the user is looking for appositives in the pattern Person Noun of Company, for example John Smith, chairman of X-Corp. ${ }^{1}$ Note that the query is not particularly linguistically sophisticated: the Position item is glossed as a noun group and the single preposition of is specified when a looser restriction (perhaps to be any preposition) would certainly turn up more results. However, this query is quick and simple to construct and can be used as a diagnostic for a more detailed query.

A more complex query is shown in Figure 5 for a protein interaction task. The sort interaction in Figure 5 could be defined as a disjunction of the constraints head word = interact, head word = bind, head word = associate).

\subsection{One-off Query vs Pattern Base}

Highlight can operate in two distinct modes: interactive and batch. The interactive mode suits one-off queries as seen but can also be used to prototype queries on a subset of a corpus. Once a user is satisfied with the accuracy of a query, this user-defined 'pattern' can be stored for later use. Batch mode is used when a query is to be run over a large amount of text and requires no intervention from the user other than the initial set-up.

\subsection{Preprocessing Files and Scalability}

If a user makes two queries over the same set of documents it does not make sense to do all the linguistic processing twice. To avoid this, documents

\footnotetext{
${ }^{1}$ We currently do not index commas but a positional constraint representing separation by a comma could easily be added.
}

can be preprocessed. Preprocessing involves tagging, chunking, recognition of sorts, and conversion of the results into a set of constraints. At query time, there is no further linguistic processing to be done, just constraint satisfaction.

The system has a relatively simple outer loop which considers a query for each document in turn (rather than e.g. using a single cross-document index). If a document has not been preprocessed, it is processed, then tested against the query. If a document has been preprocessed, the results of preprocessing are loaded, and tested against the query. Preprocessing produces a worthwhile increase in speed. Loading the preprocessed files ready for constraint satisfaction takes (on average) less than a tenth of the time it takes to process the files to get to the same stage. However, preprocessed files do take around 60 times more filespace than the source files from which they are derived ${ }^{2}$.

Although loading a preprocessed file is much faster than processing from scratch, the loading is still a significant factor in the total processing time. In fact for simple queries the loading accounts for over $90 \%$ of processing time. We were therefore keen to load only those files where there was a chance of success for a query. One way to do this is to split the query into an IR and an IE component and to use IR to pre filter the set of documents. If we only have to load one tenth of the documents then again we can expect a 10 times speed up (assuming the time to do IR is relatively trivial relative to the time as a whole).

A simple way to achieve IR filtering is to extract out any non-optional keywords from the query, and then only process those documents that contain the keywords. However, many of the queries which we use do not contain keywords at all, only sorts. In these cases, we cannot run IR over the source documents, since these do not contain the sortal information. Instead we search over a summary file which is created during preprocessing. This contains a set of all the sorts and words found in the file. The IR stage consists of selecting just those files which match the sorts and keywords in the query. This set is then passed to the IE component which deals with relational constraints such as same sentence, and interactions between constraints which have to be calculated on the fly such as precedes which are not indexed during preprocessing.

The IE component satifies the constraints in the

\footnotetext{
${ }^{2}$ This is worse than it need be: we have not yet attempted to rationalise the preprocessed files, or use encoding schemes to reduce redundancy.
} 


\section{Intelligent Search and Extraction of Information}

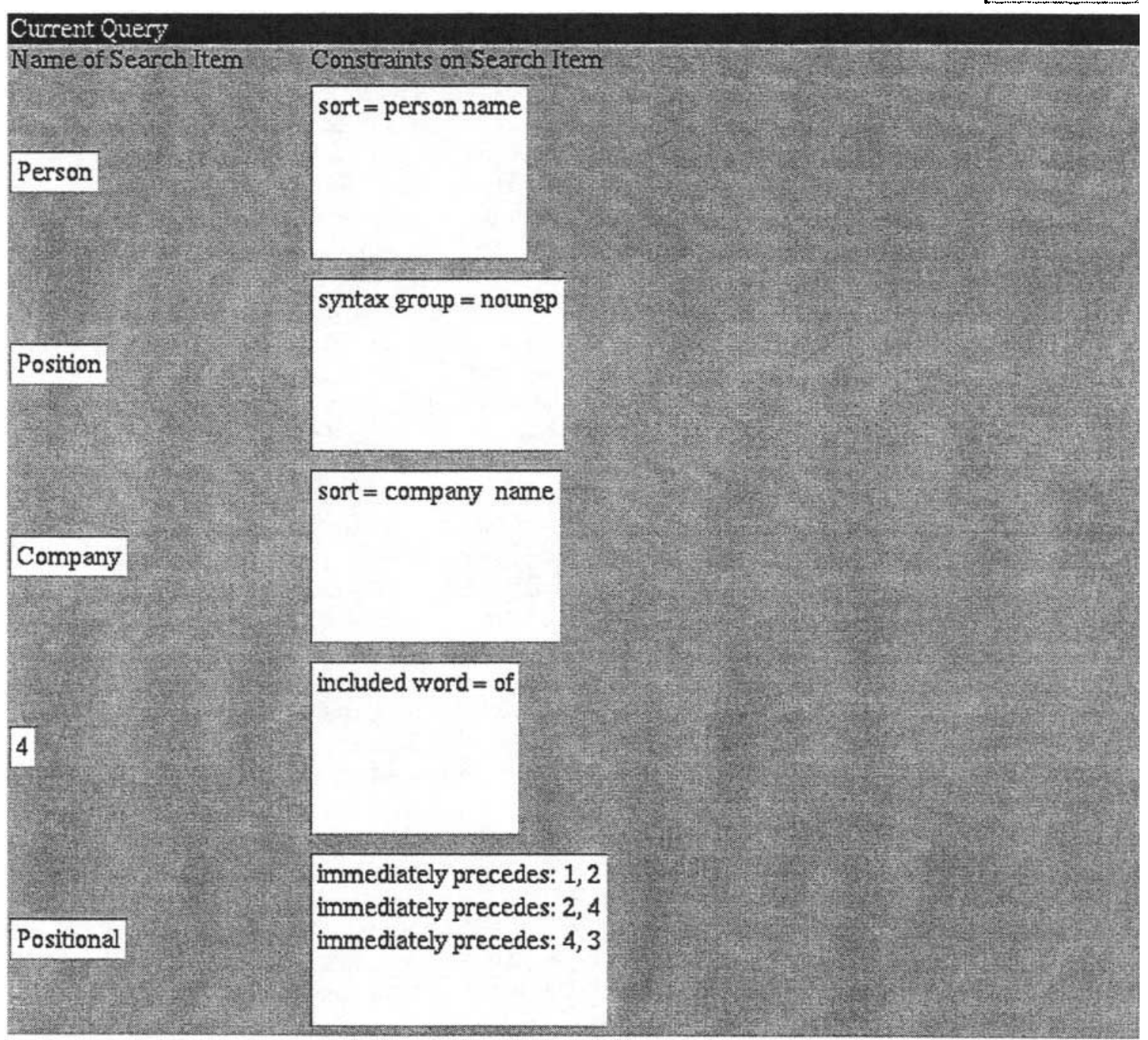

Figure 4: A typical one-off query for case: John Smith, chairman of X-Corp 


\section{Intelligent Search and Extraction of Information}

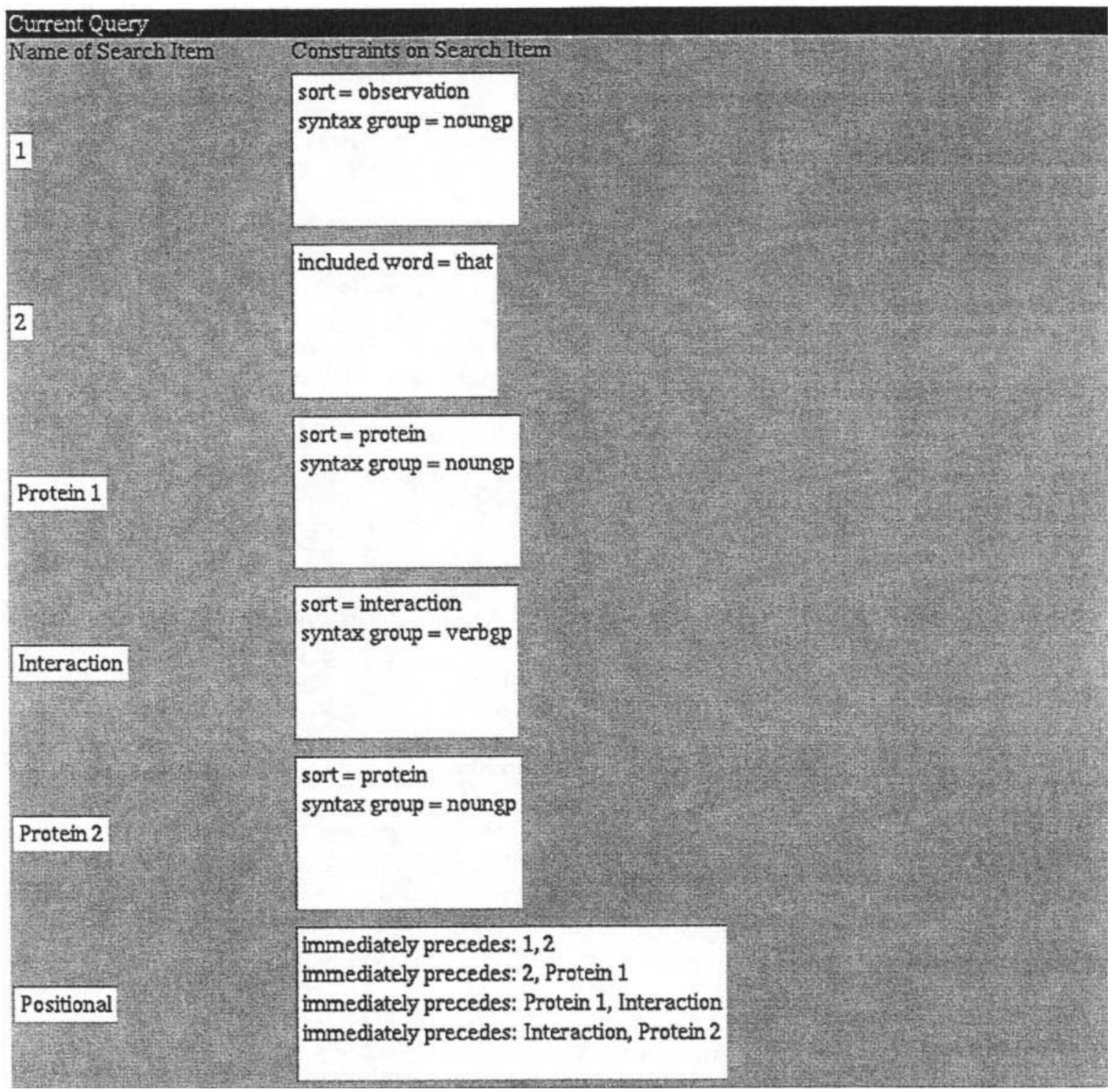

Figure 5: Query for protein interactions e.g. observe that Taf-1 binds TBP. 
query against the constraints in the preprocessed file. The constraint solver tries to satisfy the most specific constraints in the query first. Constraints are indexed and reverse indexed for efficiency.

The processing times we currently achieve are very good by the standards of Information Extraction, and are adequate for the applications for which we have been using the system. The current approach is similar to that of e.g. Molla and Hess (Aliod and Hess, 1999), who first partition the index space into separate documents, and use the IR component of queries as a filter.

Table 1 shows the difference in processing times on two queries for two different datasets. Times for processing each query on each dataset are labelled Old for Highlight with no IR and no preprocessed files, New for Highlight with preprocessed files and NewIR for Highlight with both an IR stage and preprocessed files. ${ }^{3}$

New\% and NewIR\% give New/Old and NewIR/Old respectively. From the table we can see that (for these queries) adding the preprocessed files reduces total processing time by around $75 \%$. Adding the IR stage reduces it by a further couple of percent in the case of the FT files and by $10 \%$ for the WSJ files. The performance increase on FT data is less dramatic because the data provides more hits (i.e. we extract more templates per input file) but note that for both datasets these improvements are at the worse end of the spectrum: both the WSJ and FT files stand a very good chance of containing both person and company (the sorts in our test queries) and so the IR component will propose a large number of each set for IE treatment, and we can also expect several hits per file, which tends to slow query processing. In other queries, e.g. a search for a company name, we would expect things to be much quicker as borne out by the results in Table 2.

\section{Performance}

Figures 6, 7 correspond to the queries in Figures 3 and 4 respectively. Figure 8 is the result of searching for protein interactions (a more general version of the query in Figure 5.) ${ }^{4}$ Table 2 contrasts these results. FilesIR and FilesIE refer to the number

\footnotetext{
${ }^{3}$ The WSJ dataset consists of 100 Wall Street Journal articles, the FT set comes from 60 Financial Times documents. Query 1 looks for a person and company in the same sentence while Query 2 is for Person, $X$ of/at/in Company. All tests were carried out on a Sun Ultra $2200(200 \mathrm{MHz}, 256 \mathrm{Mb}$ RAM) running Sicstus Prolog 3.7.1 under Solaris 7.

${ }^{4}$ For space reasons we have only presented a sample of our query results.
}

of files input to each stage of the system, i.e. the query in Figure 6 was run over 500 news articles, only one of them was selected by our IR component and was thus input to the IE component. Hits denotes how many of the files passed to IE actually had at least one template in them and Templates shows how many templates were extracted as a result of the query. Time is the total time for the query in seconds.

The query in Figure 6 looks for documents containing Esso and a location. ${ }^{5}$ Because Esso is such a good discriminator in this document set, appearing in only one out of 500 documents, the IE query only considers one document and the whole query succeeds in $0.5 \mathrm{sec}$.

Figure 7 takes longer than this because 218 of 500 files contain the common sorts person and company. Figure 8 is similar but has the additional overhead of around $2 / 3$ of all files having hits where Figure 7 has only 1/20.

Factors affect the total amount of time that a query takes include:

- specificity of the query

- complexity of the query

- size of the source file

- whether or not there is a hit in the file

With the addition of the IR stage, the specificity of the query has the potential to impact greatly on total query time as already seen. Having a hit in the file can also significantly affect timings since there is no chance of an early failure of constraint satisfaction. For example, taking a file from each of the queries in Figures 7 and 8 which each take $270 \mathrm{msec}$ to load (i.e. are of the same size) we find that the one in which there is no hit takes $10 \mathrm{msec}$ to process but the one is which there is a hit takes $6.5 \mathrm{sec}$ (of which just $110 \mathrm{msec}$ is spent writing results to file.)

The lessons from these results are not particularly surprising: queries should use the most specific words and sorts possible to get good value from the IR component. If there are many solutions-and remember that this system aims to extract specific information rather than a relevant document or document passage-then there will be a time penalty.

\footnotetext{
${ }^{5}$ Because there is no sentential restriction on these two items, they do not appear on the same row in the output. This is an option available to the user which means that in queries of this kind, the output is a summary of the "true" output (a cross product of each item with each other item) which can obviously result in very large output tables.
} 


\begin{tabular}{|c|c|c|c|c|c|c|}
\hline Dataset & Query & Old (msec) & New (msec) & New\% & NewIR (msec) & NewIR\% \\
\hline WSJ & 1 & 133880 & 32890 & 25 & 19120 & 14 \\
WSJ & 2 & 135840 & 33610 & 25 & 19540 & 14 \\
& & & & & & \\
FT & 1 & 94830 & 21300 & 22 & 18850 & 20 \\
FT & 2 & 92240 & 21590 & 23 & 18960 & 21 \\
\hline
\end{tabular}

Table 1: Comparison of IR and non-IR preprocessing

\begin{tabular}{|c|c|c|c|c|c|}
\hline Figure & FilesIR & FilesIE & Hits & Templates & Time (s) \\
\hline 6 & 500 & 1 & 1 & 19 & 0.5 \\
7 & 500 & 218 & 11 & 11 & 27 \\
8 & 100 & 59 & 42 & 44 & 66 \\
\hline
\end{tabular}

Table 2: Comparison of query times

Our expectation is that complex queries which also involve a lot of hits are more likely for batch mode operation, so the time penalty will not be so crucial. One-off queries will tend to be simpler, involving searches for less frequent information. However, we are also investigating further ways to improve processing speed, in particular during constraint satisfaction.

\section{Evaluation}

We have not yet performed a large-scale evaluation of the user-customisable version of Highlight. The earlier version of Highlight was evaluated for the task of extracting protein interactions, obtaining a recall of $55-58 \%$ and precision of $77 \%$. For this particular task we would not expect the results for the new system to differ significantly: the underlying engine is the same and we can use the interface to generate sets of constraints which are equivalent to the previous patterns. For example, Figures 5 (above) and 9 show how an old pattern can be generated in the new interface. Both look for phrases such as ...observed that TAF binds $T B P$, the old pattern places an interaction tag around the relevant text while in the new interface the user selects which items will be output (in this case, the ones which have names rather than numbers.)

For other tasks, there are some factors which might affect the relative recall and precision figures between the original Highlight system and the customisable version. Firstly, the original Highlight system can use cascades of pattern matching rules to perform 'blocking tactics' i.e. one rule fires for the sole purpose of preventing another from firing. To achieve a similar ability in customisable Highlight we need to introduce a way of choosing between alternative queries when more than one can apply e.g. by always choosing the most specific. Secondly, the original Highlight sometimes resorted to external program calls which is no longer possible.

\section{Applications}

The system described in this paper is still under development but is already being used in pilot projects with commercial clients. Areas in which it has been deployed include:

- expertise database: extracting relationships between employees and the projects in which they are involved from internal company documents. The relationships degrade from those which represent certain involvement (e.g. writing a report) to those that represent possible involvement (e.g. mentioned in the same sentence).

- competitor database: extracting information about companies from Financial Times documents. Here, we were after in particular the chairman, chief executive and so on of any companies in the texts. Again, sets of relationships showed the degree of certainty with which a person was related to a company.

- conference database: extracting information such as conference title, location, date and cost from conference calls downloaded from the web. This task was particularly interesting because it required the gathering of information from a variety of locations in each document rather than the more usual single sentence.

In order to test the flexibility of the new system, we have also been recoding previous applications of the Highlight system. 


\begin{tabular}{|c|c|c|c|c|}
\hline \multicolumn{5}{|c|}{ Query results } \\
\hline Source & 1 & 2 & Sentence & Link \\
\hline wsj_0473 & $\begin{array}{l}\text { Esso Resources } \\
\text { Canada Ltd. }\end{array}$ & - & $\begin{array}{l}\text { The producers inchude Shell Canada Ltd., a unit of } \\
\text { Royal Dutch / Shell Group; Esso Resources Canada } \\
\text { Ltd., a unit of Imperial Oil Ltd., which is } 71 \\
\text { \%-owned by Exxon Corp.; and Gulf Canada } \\
\text { Resources Ltd., a unit of Olympia \& York } \\
\text { Developments Ltd. }\end{array}$ & link \\
\hline wsj_0473 & - & the Arctic & $\begin{array}{l}\text { "Foothills wants to make it clear to other pipeline } \\
\text { companies that it's on first insofar as transporting } \\
\text { gas from the Arctic to southern markets," Mr. } \\
\text { Hillary said. }\end{array}$ & link \\
\hline wsj_0473 & - & Canada & $\begin{array}{l}\text { At least two rival applications are expected to } \\
\text { emerge in coming months, including one from } \\
\text { TransCanada PipeLines Ltd., Canada's largest } \\
\text { natural gas pipeline operator. }\end{array}$ & link \\
\hline wsj_0473 & - & Houston & $\begin{array}{l}\text { The Toronto-based company, together with } \\
\text { Tenneco Inc. of Houston, has had an incomplete } \\
\text { proposal filed with Canadian regulators since } 1984 \\
\text { that it is now updating. }\end{array}$ & link \\
\hline wsj_0473 & 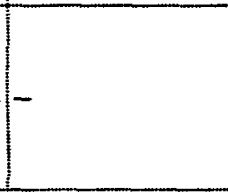 & $\begin{array}{l}\text { the Mackenzie } \\
\text { River delta }\end{array}$ & $\begin{array}{l}\text { Like Foothills, TransCanada's Polar Gas } \\
\text { consortium plans to build a pipeline directly south } \\
\text { from the Mackenzie River delta in Canada' } \\
\text { western Arctic with an initial capacity to transport } \\
1.2 \text { billion cubic feet of gas daily. }\end{array}$ & link \\
\hline wsi_0473 & - & Alberta & $\begin{array}{l}\text { Industry sources said they expect a fierce battle to } \\
\text { emerge between TransCanada, which has a } \\
\text { monopoly on Canadian gas transportation east of } \\
\text { Alberta, and Nova and Westcoast, which control } \\
\text { the pipelines within and nunning west of Alberta, } \\
\text { respectively. }\end{array}$ & link \\
\hline wsj_0473 & - & $\begin{array}{l}\text { the Prudhoe Bay } \\
\text { area }\end{array}$ & $\begin{array}{l}\text { U. S. gas buyers must also decide whether they want } \\
\text { to enter firm contracts for Mackenzie delta gas or } \\
\text { derelop Alaskan reserves in the Prudhoe Bay area } \\
\text { first, a project that has been on hold for more than } \\
\text { a decade. }\end{array}$ & link \\
\hline wsi_0473 & - & Alaska & $\begin{array}{l}\text { But Foothills said it plans to seek regulatory } \\
\text { approval to build an alternative line, the Alaska } \\
\text { Natural Gas Transportation System further north } \\
\text { toward Alaska. }\end{array}$ & link \\
\hline
\end{tabular}

Figure 6: Sample of results from query for keyword Esso and sort location 
Query results

\begin{tabular}{|c|c|c|c|c|c|c|}
\hline Source & Person & Position & Company & 4 & Sentence & Link \\
\hline wsi_0111 & $\begin{array}{l}\text { Robert L } \\
\text { Bernstein }\end{array}$ & $\begin{array}{l}\text { chairman and } \\
\text { president }\end{array}$ & $\begin{array}{l}\text { Random House } \\
\text { Inc. }\end{array}$ & of & $\begin{array}{l}\text { START Robert L Bernstein, } \\
\text { chairman and president of Random } \\
\text { House Inc., announced his } \\
\text { resignation from the publishing } \\
\text { house he has run for } 23 \text { years. }\end{array}$ & link \\
\hline wsj_0209 & $\begin{array}{l}\text { Murray } \\
\text { Robinson }\end{array}$ & president & $\begin{array}{l}\text { Delta \& Pine } \\
\text { Land } \mathrm{Co} \text {. }\end{array}$ & of & $\begin{array}{l}\text { " The development could have a } \\
\text { dramatic effect on farm } \\
\text { production, especially cotton, , said } \\
\text { Murray Robinson, president of } \\
\text { Delta \& Pine Land Co., a } \\
\text { Southwide Inc. subsidiary that is } \\
\text { one of the largest cotton seed } \\
\text { producers in the U.S. }\end{array}$ & link \\
\hline wyj_0276 & Michael Blair & $\begin{array}{l}\text { former president } \\
\text { and chief } \\
\text { executive officer }\end{array}$ & Enfield Corp. & of & $\begin{array}{l}\text { START Michael Blair, former } \\
\text { president and chief executive } \\
\text { officer of Enfield Corp., failed to } \\
\text { win election to the company's } \\
\text { board at a special shareholder } \\
\text { meeting. }\end{array}$ & link \\
\hline wss_0391 & $\begin{array}{l}\text { David } \\
\text { Rockefeller }\end{array}$ & chairman & Rockefeller Group & of & $\begin{array}{l}\text { David Rockefeller, chairman of } \\
\text { Rockefeller Group, said the } \\
\text { company talked with many } \\
\text { potential investors in Japan, the } \\
\text { United States and Europe. }\end{array}$ & link \\
\hline wsj_0509 & Stephen Wolf & chairman & UAL Inc. & lof & $\begin{array}{l}\text { Among the other alumni are } \\
\text { Stephen Wolf, now chairman of } \\
\text { UAL Inc., and Thomas Plaskett, } \\
\text { president of Pan Am Corp }\end{array}$ & link \\
\hline Wsi_0593 & $\begin{array}{l}\text { Joseph L } \\
\text { Dionne }\end{array}$ & $\begin{array}{l}\text { chairman and } \\
\text { chief executive } \\
\text { officer }\end{array}$ & McGraw-Hill Inc. & & $\begin{array}{l}\text { START Joseph L. Dionne, } \\
\text { chairman and chief executive } \\
\text { officer of McGraw-Hill Inc., was } \\
\text { elected to the board of directors of } \\
\text { this electronics manufacturer. }\end{array}$ & ఏnk \\
\hline
\end{tabular}

Figure 7: Sample of results from query for Person, $X$ of Company 
Query results

\begin{tabular}{|c|c|c|c|c|c|}
\hline Source & Protain 1 & Interaction & Protein 2 & Sentence & Link \\
\hline bio_10004 & TRRAP & interacts & $\begin{array}{l}\text { the E2F1 transactivation } \\
\text { domain }\end{array}$ & $\begin{array}{l}\text { TRRAP also interacts specifically with } \\
\text { the E2F1 transactivation domain. }\end{array}$ & link \\
\hline bio_1014 & Cdi1 & interacts & cyclin-dependent kinases & $\begin{array}{l}\text { In yeast, Cdi1 interacts with } \\
\text { cyclin-dependent kinases, inchuding } \\
\text { human CDC2 (116940), CDK2 } \\
\text { (116953), and CDK3 (123828), but not } \\
\text { with CDK4 (123829). }\end{array}$ & link \\
\hline bio_10187 & Ptrf & interacts & both TTF 1 and Pol I & $\begin{array}{l}\text { Jansa et al. (1998) demonstrated that } \\
\text { Putf interacts with both TTF1 and Pol I, } \\
\text { and binds to transcripts containing the } \\
\text { 3-prime end of pre-rRNA in vitro. }\end{array}$ & link \\
\hline bio_10261 & CNK & interacts & RAF & $\begin{array}{l}\text { CNK physically interacts with RAF and } \\
\text { appears to localize to cell-cell contact } \\
\text { regions. }\end{array}$ & link \\
\hline bio_10408 & $\begin{array}{l}\text { The SGT } \\
\text { protein }\end{array}$ & interacts & $\begin{array}{l}\text { the parvovinus } \\
\text { nonstructural protein } \\
\text { NS1 }\end{array}$ & $\begin{array}{l}\text { record (603419) The SGT protein } \\
\text { interacts with the parvovinus } \\
\text { nonstructural protein NS1. }\end{array}$ & link \\
\hline bio_10444 & RICK & interacts & CLARP & $\begin{array}{l}\text { Inohara et al. (1998) demonstrated that } \\
\text { RICK physically interacts with CLARP, } \\
\text { a caspase-like molecule known to bind } \\
\text { to FADD and caspase-8. }\end{array}$ & link \\
\hline bio_10876 & himan & interacts & Drosophila & $\begin{array}{l}\text { Sangoram et al. (1998) demonstrated } \\
\text { that human TIM interacts with } \\
\text { Drosophila per, mouse PER1, and mouse } \\
\text { PER2 (see 603426) in vitro. }\end{array}$ & link \\
\hline bio_11073 & MIZ1 & interacts & MYC and NMYC & $\begin{array}{l}\text { MIZ1 interacts specifically with MYC } \\
\text { and NMYC (164840). }\end{array}$ & link \\
\hline
\end{tabular}

Figure 8: Sample of results from query for $X$ interacts with $Y$

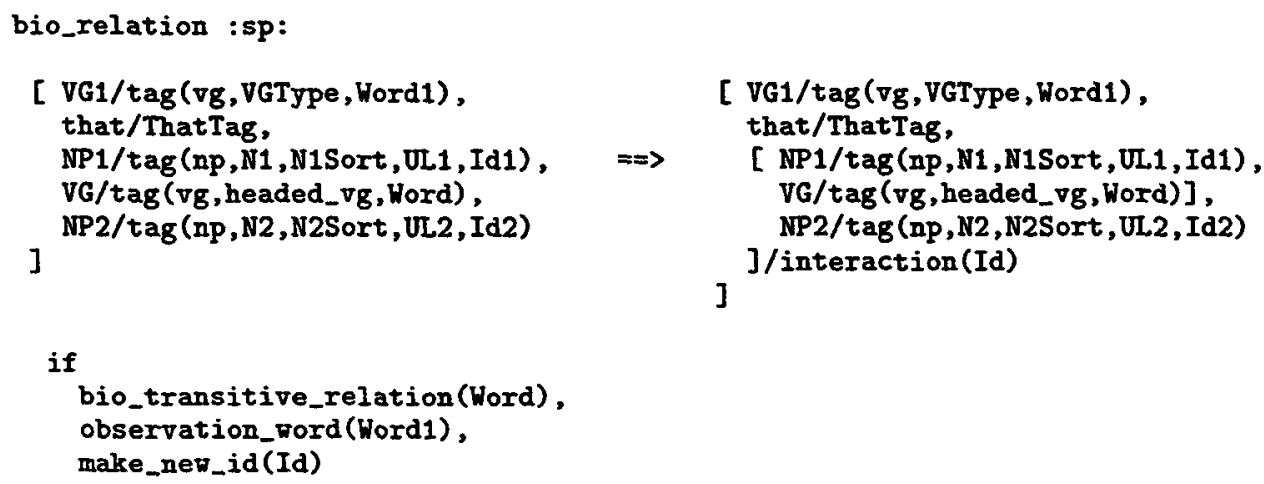

Figure 9: Pattern from basic Highlight for case: observe that Taf-1 binds TBP. 


\section{Summary}

This paper has given a brief outline of a system which may be used for one-off queries in the style of current Information Retrieval systems but with the advantages of Information Extraction technology. The same interface can be used to build queries appropriate for more traditional batch mode Information Extraction tasks.

\section{References}

Diego Molla Aliod and Micheal Hess. 1999. On the scalability of the answer extraction system "extrans". In Applications of Natural Language to Information Systems (NLDB'99), pages 219224.

Claire Cardie and David Pierce. 1998. Proposal for an interactive environment for Information Extraction. Technical Report TR98-1702, Department of Computer Science, Cornell University.

Jerry R. Hobbs, Douglas Appelt, David Israel John Bear, Megumi Kameyama, Mark Stickel, and Mabry Tyson. 1996. Fastus: A cascaded finite-state transducer for extracting information from natural-language text. In E. Roche and Y. Schabes, editors, Finite State Devices for Natural Language Processing. MIT Press.

David Milward. 2000. Distributing representation for robust interpretation of dialogue utterances. In Proceedings of the 38th ACL Conference.

Ellen Riloff. 1993. Automatically constructing a dictionary for Information Extraction tasks. In Proceedings of the 11th National Conference on Artificial Intelligence, pages 811-816. AAAI/MIT Press.

Ellen Riloff. 1996. Automatically generating extraction patterns from untagged text. In Proceedings of the 13th National Conference on Artificial Intelligence, pages 1044-1049.

Stephen Sonderland, David Fisher, Jonathan Aseltine, and Wendy Lehnert. 1995. CRYSTAL:inducing a conceptual dictionary. In Proceedings of the 14th International Joinct Conference on Artificial Intelligence.

Rohini Srihari and Wei Li. 1999. Information extraction supported question answering. In Proceedings of the Eighth Text Retrieval Conference (TREC-8).

James Thomas, David Milward, Christos Ouzounis, Stephen Pulman, and Mark Carroll. 2000. Automatic extraction of protein interactions from scientific abstracts. In Pacific Symposium on Biocomputing.
Text Retrieval Conferences (TREC). 2000. http://trec.nist.gov/.

Roman Yangarber and Ralph Grishman. 1997. Customization of Information Extraction systems. In Proceedings of the International Workshop on Lexically Driven Information Extraction. 